\title{
The Influence of Visual Perturbations on the Neural Control of Limb Stiffness
}

\author{
Jeremy Wong, ${ }^{1,2}$ Elizabeth T. Wilson, ${ }^{1,2}$ Nicole Malfait, ${ }^{1,4}$ and Paul L. Gribble ${ }^{1,3}$ \\ ${ }^{1}$ Department of Psychology, ${ }^{2}$ Graduate Program in Neuroscience, and ${ }^{3}$ Department of Physiology and Pharmacology, The University \\ of Western Ontario, London, Ontario, Canada; and ${ }^{4}$ Unité Mixte de Recherche 6149, Neurosciences Intégratives et Adaptatives, \\ Centre National de la Recherche Scientifique, Marseille, France
}

Submitted 17 March 2008; accepted in final form 27 July 2008

\begin{abstract}
Wong J, Wilson ET, Malfait N, Gribble PL. The influence of visual perturbations on the neural control of limb stiffness. $J$ Neurophysiol 101: 246-257, 2009. First published July 30, 2008; doi:10.1152/jn.90371.2008. To adapt to novel unstable environments, the motor system modulates limb stiffness to produce selective increases in arm stability. The motor system receives information about the environment via somatosensory and proprioceptive signals related to the perturbing forces and visual signals indicating deviations from an expected hand trajectory. Here we investigated whether subjects modulate limb stiffness during adaptation to a purely visual perturbation. In a first experiment, measurements of limb stiffness were taken during adaptation to an elastic force field (EF). Observed changes in stiffness were consistent with previous reports: subjects increased limb stiffness and did so only in the direction of the environmental instability. In a second experiment, stiffness changes were measured during adaptation to a visual perturbing environment that magnified hand-path deviations in the lateral direction. In contrast to the first experiment, subjects trained in this visual task showed no accompanying change in stiffness, despite reliable improvements in movement accuracy. These findings suggest that this sort of visual information alone may not be sufficient to engage neural systems for stiffness control, which may depend on sensory signals more directly related to perturbing forces, such as those arising from proprioception and somatosensation.
\end{abstract}

\section{N T R O D U C T I O N}

The human nervous system can modify the mechanical behavior of the limb to adapt to task requirements for both posture and movement. By controlling the level of antagonist muscle coactivation, the nervous system can adjust mechanical impedance about a joint and compensate for perturbations resulting from limb dynamics and external forces (Hogan 1985). Arm stiffness is modulated to compensate for movement environments that feature unstable loads. Burdet et al. (2001) showed that stiffness was increased only in the direction of an environmental instability, suggesting that stiffness can be modulated to efficiently increase limb stability. Reaching movements with higher levels of stiffness (Burdet et al. 2001; Shiller et al. 2002) and muscle coactivation (Gribble et al. 2003; Osu et al. 2004) are also associated with decreased movement variability and reaches to smaller visual reaching targets. This suggests that stiffness may be used to increase movement accuracy. It has also been suggested that increased stiffness may reduce the perturbing effects of neuromuscular noise (Osu et al. 2004; Seidler-Dobrin et al. 1998; Selen et al. 2006a,b).

Much of the existing work on the neural control of arm stiffness has been done in the context of unstable force envi-

Address for reprint requests and other correspondence: P. L. Gribble, Department of Psychology, The University of Western Ontario, 1151 Richmond St., London, Ontario, Canada N6A 5C2 (E-mail: pgribble@uwo.ca). ronments (e.g., negative elastic force fields) imposed on the limb by robotic devices (Burdet et al. 2001; Franklin et al. 2003, 2004, 2007a,b). In these tasks, subjects received both visuals, and proprioceptive and somatosensory information about the perturbing force environment. Somatosensory signals from cutaneous receptors and proprioceptive signals from afferents such as muscle spindles and Golgi tendon organs may provide information about the magnitude and direction of perturbing forces. In contrast, vision of the limb provides information about position and thus can provide only indirect information about perturbing forces, to the extent that deviations from an expected position are related to force perturbations. Thus although changes in stiffness have been observed in response to both force perturbations and changes to visual task demands, only one study has been reported examining the relative importance of visual versus somatosensory and proprioceptive information for the neural control of arm stiffness (Franklin et al. 2007b).

Here we tested whether stiffness modulation would occur in response to a purely visual perturbation that approximated the visual information associated with an unstable force environment. We compared the extent of stiffness modulation in an unstable force environment to that in a second environment defined by a purely visual perturbation. Stiffness was measured midway through movement, using a position-servo control method reported previously (Darainy et al. 2007). In a first experiment, the goal was to establish, using this method, a selective increase in stiffness similar to that reported by Burdet et al. (2001) during training in an elastic force field. In a second experiment subjects performed reaching movements in a novel environment that was designed as a visual analog of the elastic force field. Visual feedback of the hand was manipulated to magnify deviations made perpendicular to movement direction. In a third experiment we more precisely matched the visual perturbations of the two environments such that differences between stiffness resulting from visual and force training could be more directly compared.

Subjects who trained in the elastic force field showed significant increases in arm stiffness only in the direction of perturbing forces. In contrast, subjects trained in the visual gain environments showed no accompanying change in stiffness, despite reliable improvements in movement accuracy. These findings suggest that this sort of visual information alone may not be sufficient to engage neural systems for stiffness control, which may depend in part on afferent signals more closely related to environmental forces. In the absence of these signals,

\footnotetext{
The costs of publication of this article were defrayed in part by the payment of page charges. The article must therefore be hereby marked "advertisement" in accordance with 18 U.S.C. Section 1734 solely to indicate this fact.
} 
subjects may use other strategies to improve movement accuracy.

\section{METHODS}

\section{Subjects}

Twenty-five right-handed subjects (eight females) between 17 and $35 \mathrm{yr}$ of age (mean age $=20.1 \mathrm{yr}$ ) were randomly assigned to three experimental groups. Subjects reported no history of visual, neurological, or musculoskeletal disorder. Written informed consent was obtained from each subject prior to participation. The UWO Research Ethics Board approved all procedures.

\section{Apparatus}

Subjects performed reaching movements while grasping the handle of an InMotion ${ }^{2}$ robotic linkage (InMotion Technologies, Cambridge, MA) in the right hand. A six-axis force transducer (ATI Industrial Automation, Apex, NC; resolution: $0.05 \mathrm{~N}$ ) located inside the handle, measured forces at the hand. Movements were made in a horizontal plane along the surface of a desk, at shoulder height (see Fig. 1A). A custom-built air sled was used to support the subject's arm against gravity while maintaining minimal levels of friction between the air sled and desk. Shoulder straps were used to maintain the subject in a seated position, keeping the shoulder in place and minimizing trunk movements. The wrist was braced as well, restricting movements to shoulder and elbow rotations. The subjects' view of their arm was occluded by a mirror placed just above the shoulder. Visual feedback of hand position was provided on this mirror in real time using a computer-controlled liquid crystal display projector.

\section{Movement task}

Subjects were asked to move their limb from a start target to an end target, both presented visually as mentioned earlier, as filled circles (diameter $=25 \mathrm{~mm}$ ). The start target was positioned $5 \mathrm{~cm}$ away from the subject's torso, along the subject's midline. The end target was located $35 \mathrm{~cm}$ away from the start target, also along the midline. Subjects were instructed to move their limb in a straight, smooth fashion within a fixed duration of $1,200 \pm 75 \mathrm{~ms}$. A cursor (a small filled circle, diameter $=8 \mathrm{~mm}$ ) was displayed in real time to represent the position of the hand. Feedback was given to the subject on a trial-by-trial basis about movement speed and also when movements strayed laterally by $>2 \mathrm{~cm}$. Each experiment consisted of 11 blocks of 40 movements (440 movements total).

A first group of subjects $(n=10 ; 3$ females; mean age $=21.6 \mathrm{yr})$ trained in a lateral elastic force field $\left(\mathrm{EF}_{\text {strong }}\right)$. The goal was to

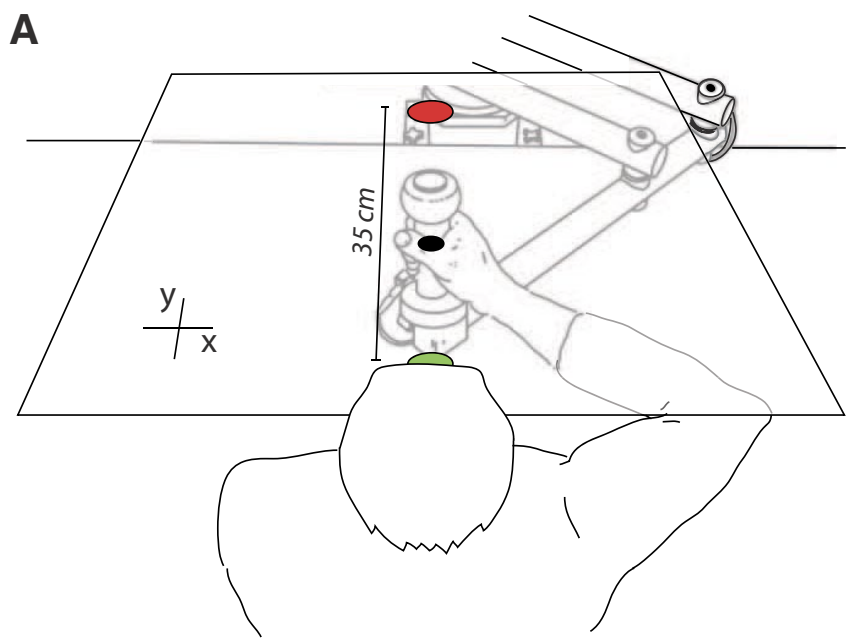

demonstrate- using our apparatus and method for estimating stiffness-a selective increase in stiffness similar to that reported by Burdet et al. (2001). The force field was defined by

$$
\left[\begin{array}{l}
F x \\
F y
\end{array}\right]=k\left[\begin{array}{l}
x \\
0
\end{array}\right]
$$

where $F x$ and $F y$ (Newtons) represent forces exerted by the robot on the hand in the lateral and forward/backward directions, respectively, and $x$ (meters) represents the lateral distance of the hand from a line connecting the start and end targets. The constant $k$ was set to 250 $\mathrm{N} / \mathrm{m}$ for all subjects in the $\mathrm{EF}_{\text {strong }}$ group.

A second group of subjects $(n=10 ; 3$ females; mean age $=18.7$ yr) trained in a visual analog of the elastic force field, which we refer to as a visual gain field (VGF). Here, no forces were applied to the hand but visual feedback of hand position was manipulated using a positive gain so as to magnify the visual lateral distance of the hand from the midline. Specifically, we used a gain of 2.0 so that the distance of the visual cursor away from the midline was twice the actual distance of the hand from midline. Thus whereas in the elastic force field the hand was physically pushed in a lateral direction in proportion to lateral deviation from a straight line, in the visual gain field no forces were applied by the robot but, rather, the visual feedback was manipulated to visually magnify lateral deviations of the hand from a straight line.

A third group of subjects $\mathrm{EF}_{\text {weak }}(n=5 ; 2$ females; mean age $=$ $19.9 \mathrm{yr}$ ) experienced a similar force field as subjects in the $\mathrm{EF}_{\text {strong }}$ group, but forces were reduced $(k=125 \mathrm{~N} / \mathrm{m})$ so that the initial lateral hand path deviations before training matched those observed by subjects in the VGF group.

For all three groups, subjects initially performed 160 movements (four blocks of 40 movements) in a baseline environment, in which neither the force nor the visual perturbations were applied. Following this, subjects performed 280 movements (seven blocks of 40 movements) in the elastic force or visual gain field.

In all cases adaptation was quantified by changes in movement accuracy over the course of training. To characterize movement accuracy we measured the mean perpendicular distance (mPD) over the middle portion of movement. The mPD was defined as the mean absolute perpendicular deviation made from a straight line connecting the start and end targets over the middle $2 \mathrm{~cm}$ of movement (see Fig. $1 B$ ). The location of this measure was selected to coincide with the location of stiffness measurement (see following text). Individual scores were averaged across bins of 10 movements, except during the initial learning stages, where the first 5 single movements are reported separately.

Statistical analysis of kinematic differences were assessed using split-plot ANOVA (group $\times$ training) and post hoc tests.

B

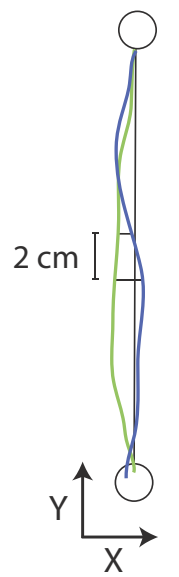

FIG. 1. Experimental apparatus. A: subjects performed seated reaching movements while grasping the handle of the robotic device. Vision of the limb was occluded; $35-\mathrm{cm}$ movements were made along the subject's midline beginning (in green) 5 $\mathrm{cm}$ away from the body. $B$ : accuracy was characterized by measuring the mean absolute perpendicular deviation in the center of the movement. Two sample movements are shown in blue and green. 


\section{Stiffness estimation during movement}

Estimation of endpoint stiffness was made using position-servo control using a method reported previously by Darainy et al. (2007). Briefly, stiffness was estimated by perturbing the hand a specified distance away from an on-line estimate of the trajectory the hand would have taken during an unperturbed movement, and measuring the restoring forces at the robot handle (as well as taking account of forces expected due to limb dynamics). A detailed description of the procedure is presented in APPENDIX A.

Perturbations occurred on randomly selected trials at a rate of $20 \%$. This value was chosen to coincide with other studies (Darainy et al. 2004, 2006, 2007; Lametti et al. 2007) and to balance the need for an adequate number of stiffness measurements with the potential of influencing subject's behavior. Stiffness was estimated both at the end of the baseline (block 4) and learning conditions (block 11). All stiffness estimation trials were run in the absence of any force field. Subjects were instructed not to respond in any way to the perturbations. Visual feedback of the hand was removed during stiffness measurement perturbations. Perturbations lasted $500 \mathrm{~ms}$ and force signals were estimated during a 50-ms time window that was selected on a per-trial basis about $250-300 \mathrm{~ms}$ after the perturbation onset, at which point the restoring forces were judged to be stable (e.g., see Fig. $2 C$ ). This delay between perturbation start and restoring force estimation was necessary to ensure that the hand was moved the full distance away, and that force signals had stabilized, and is similar to times reported in other recent studies of stiffness during movement (Darainy et al. 2007; Gomi and Kawato 1996, 1997).

Twelve-millimeter $(12.0 \pm 0.1 \mathrm{~mm})$ position-servo perturbations were applied in eight directions spanning $360^{\circ}(0,45,90,135, \ldots$, $315^{\circ}$ ). Stiffness was estimated using a total of 24 measurements, 3 in each of the eight directions. Coefficients for the robot's position-servo controller were set at $6,000 \mathrm{~N} / \mathrm{m}$ and $240 \mathrm{Ns} / \mathrm{m}$, respectively. Positions of the robot handle and robot control signals were sampled and commanded, respectively, at $600 \mathrm{~Hz}$.

\section{Example stiffness measurement}

Figure 2 shows an example of a typical position servo, in this case made toward the body - that is, in the direction opposing movement. The window over which restoring forces were measured is indicated by gray vertical lines. Figure $2 A$ shows the major axis of movement. The perturbation is shown in red. The change in position $(\mathrm{d} x)$ is shown in Fig. $2 B$. Note that the positional perturbation is smooth and stable over the hold phase of the perturbation. Measured forces at the handle are shown in Fig. $2 C$, illustrating the smooth and stable force signals we observed during the estimation window. Figure $2 D$ shows a


FIG. 2. Representative position servo. A: an example position servo displacement, shown in red, made in the negative direction, opposite to movement direction. $B$ : changes in position during the perturbation. Note the change in $\mathrm{Y}$ in the negative direction (red) and no change in $\mathrm{X}$ (blue). $C$ : force signals during perturbation. $D$ : a set of position displacements for one stiffness measurement and the corresponding restoring forces, in $E$. 
collection of $24 \mathrm{~d} x$ measures, resulting from three perturbations in each of eight directions. Note that the positional servos are realized with very high accuracy and little variability. Figure $2 E$ shows the measured $\mathrm{d} F$ values. Note that $\mathrm{d} F$ values cluster according to the direction of the applied perturbation and the magnitudes are opposite to the positional perturbation. Leftward perturbations result in restoring forces measured to the right and vice versa. This provides some additional confidence that the position servos and the estimates of $\mathrm{d} x$ and $\mathrm{d} F$ were reasonable. In addition we carried out cross-validation tests to assess the magnitude of errors in the estimation of the hand position (see APPENDIX A). APPENDIX B describes a number of other controls to test the sensitivity of stiffness estimates.

\section{R E S U L T S}

Experiment 1: selective increase in stiffness in a elastic force field

Subjects in the elastic field $\mathrm{EF}_{\text {strong }}(n=10)$ first performed reaching movements in a baseline condition (no forces) and subsequently trained in the elastic force field. Movements were very straight at baseline $(\mathrm{mPD}=5.01 \pm 0.47 \mathrm{~mm})$ and were subsequently highly perturbed during initial exposure to the elastic field (mean PD $=22.8 \pm 2.84 \mathrm{~mm}$ ). Through training, subjects learned to attenuate the effects of the force field and performed straighter reaches by the end of training (mPD = $13.2 \pm 4.2 \mathrm{~mm}$ ). Figure $3 A$ shows changes in $\mathrm{mPD}$ across training.

To assess how limb stiffness changed in response to exposure to the elastic force field, stiffness was estimated in both the baseline period and at the end of elastic field training. Table 1 shows stiffness matrices for each $\mathrm{EF}_{\text {strong }}$ subject. Mean $R^{2}$ values representing how well the estimated stiffness matrices fit the measured force data were 0.88 for baseline and 0.89 post-EF training. The left column shows stiffness matrices for movements in the baseline condition (no forces) and the right column shows stiffness matrices after subjects trained in the elastic field. Note that for every subject, $K_{x, x}$ (stiffness in $x$ for perturbations in $x$ ), was greater following exposure to the elastic field. A MANOVA was performed to test for statistically reliable changes to elements of the stiffness matrix before versus after elastic field training. The omnibus MANOVA test for $\mathrm{EF}_{\text {strong }}$ subjects reached significance $[F(1,18)=22.37$; $P<0.001]$; post hoc tests showed that the only statistically significant difference was component $K_{x, x}[F(1,18)=51.07$; $P<0.001$; stiffness in the same direction as the elastic force field].

Figure $4 A$ shows stiffness ellipses for each $\mathrm{EF}_{\text {strong }}$ subject. Baseline stiffness is represented in black; stiffness after $\mathrm{EF}_{\text {strong }}$ training is shown in blue. Clear changes in the magnitude of stiffness can be seen in the lateral direction. Figure $5 \mathrm{~A}$ shows mean values across subjects of the four elements of the stiffness matrix. A clear change in $K_{x, x}$ is observed, in the absence of changes in other elements of the stiffness matrix.

\section{Experiment 2: visual gain condition}

A second group of subjects $(n=10)$ trained in a visual gain field (VGF subjects). Subjects' movements were initially visually perturbed, showing large apparent deviations in response to the increase in visual gain in the lateral direction. Deviations of observed (visually perturbed) hand position for the first
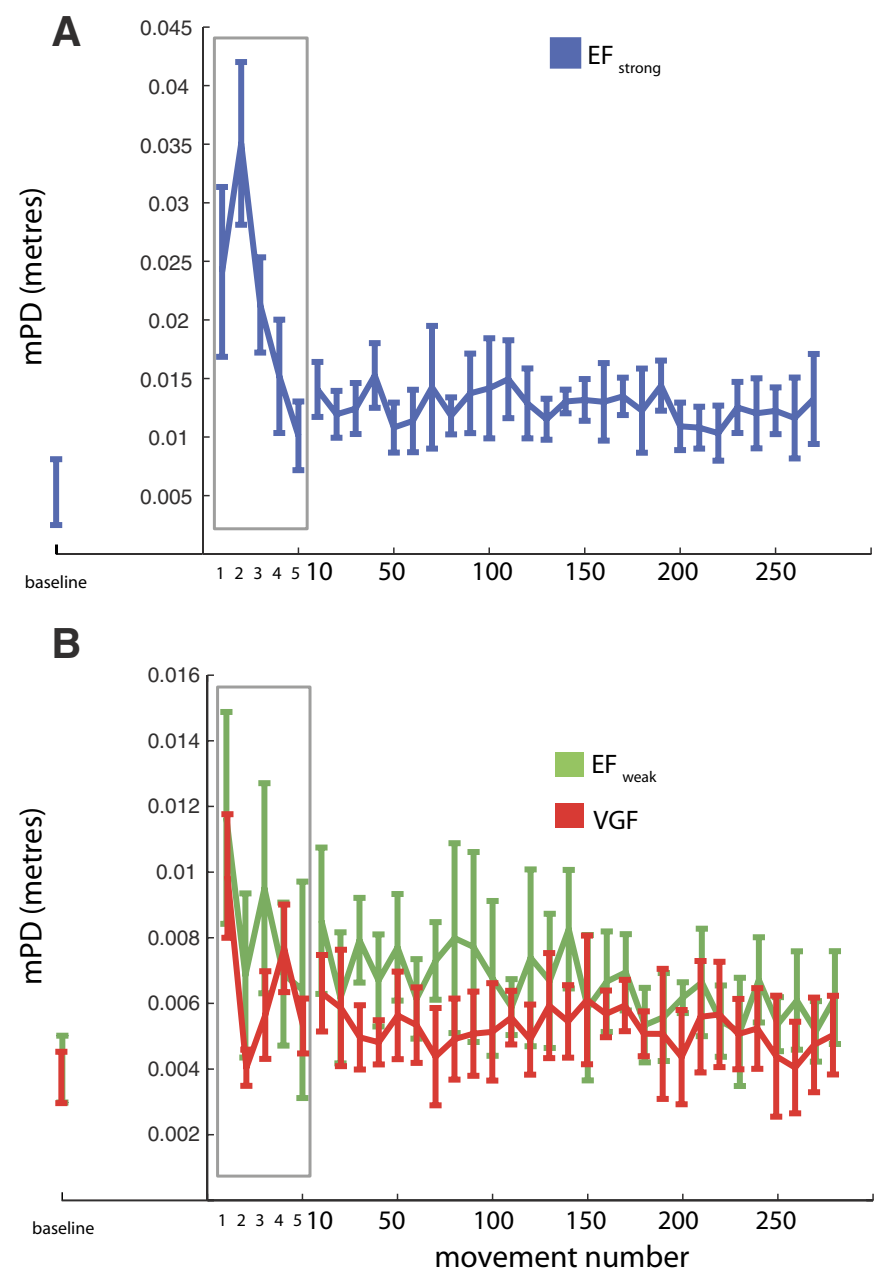

FIG. 3. Performance across training. Mean absolute deviation in the middle of movements (mean perpendicular distance [mPD]), as a function of movement number, for electric force field $(\mathrm{EF})$ subjects $(A)$ and visual gain field (VGF) and $\mathrm{EF}_{\text {weak }}$ subjects $(B)$. The gray box highlights movements 161-165 (the first 5 movements after 4 blocks of baseline trials), which are averaged across single movements, and depict initial performance in the divergent field; all other data points represent averages across 10 movements. Vertical bars represent 1SE. Note that baseline errors for all groups and errors for the $\mathrm{EF}_{\text {strong }}$ and $\mathrm{EF}_{\text {weak }}$ conditions reflect actual hand position; errors in the VGF condition reflect observed hand position, in which lateral deviations have been amplified by a factor of 2 (see METHODS).

movement in the visual gain field were initially $9.28 \pm 2.76$ $\mathrm{mm}$ and were significantly larger than $\mathrm{mPD}$ in the baseline condition $[4.34 \pm 1.17 \mathrm{~mm} ; F(1,18)=10.62 ; P=0.01]$. By the last 10 trials of training, observed $\mathrm{mPD}$ was reduced to $4.78 \pm 1.74 \mathrm{~mm}$. Associated with this visual error was a physical deviation of the hand, hidden from subjects' view. The magnitude of this deviation was half as large, or $2.39 \mathrm{~mm}$ (given the visual gain of 2.0). Mean physical hand deviation in the baseline condition prior to VGF training was $4.34 \mathrm{~mm}$; thus accuracy was increased by $44.9 \%$ following training in the $\operatorname{VGF}[F(1,18)=19.82 ; P=0.002]$. Figure $3 B$ shows changes in $\mathrm{mPD}$ across training for VGF subjects. Clear increases in movement accuracy over training can be seen.

Table 2 shows stiffness matrices for each VGF subject. No consistent changes in stiffness were observed. Figure $4 B$ shows stiffness ellipses for each VGF subject; baseline stiffness is shown in black and stiffness following VGF training is shown 
TABLE 1. Stiffness matrices before and after training in an elastic force field ( $\left.E F_{\text {strong }}\right)$

\begin{tabular}{|c|c|c|c|c|c|c|c|c|}
\hline \multirow{4}{*}{$\frac{\text { Subject }}{1}$} & & & \multicolumn{2}{|c|}{ Format for Entries } & & & & \\
\hline & & & \multirow{2}{*}{$\begin{array}{l}K_{x, x} \pm \mathrm{SE} \\
K_{y, x} \pm \mathrm{SE}\end{array}$} & \multirow{2}{*}{$\begin{array}{l}K_{x, y} \pm \mathrm{SE} \\
K_{y, y} \pm \mathrm{SE}\end{array}$} & & & & \\
\hline & & & & & & & & \\
\hline & -260.730 & 20 & 38.561 & 13 & -544.500 & 33 & 87.493 & 24 \\
\hline & 11.965 & 22 & -64.645 & 18 & -17.100 & 24 & -45.144 & 16 \\
\hline 3 & -32.875 & 17 & -93.833 & 15 & -61.410 & 30 & -48.862 & 27 \\
\hline \multirow[t]{2}{*}{4} & -201.870 & 17 & 56.742 & 11 & -536.010 & 49 & 120.610 & 50 \\
\hline & 17.181 & 14 & -44.367 & 11 & -18.531 & 53 & -73.824 & 49 \\
\hline \multirow[t]{2}{*}{5} & -157.200 & 22 & 13.200 & 22 & -386.920 & 33 & 75.421 & 29 \\
\hline & -42.818 & 23 & -66.120 & 23 & -52.310 & 37 & -54.075 & 38 \\
\hline 6 & -214.660 & 13 & 86.388 & 20 & -610.120 & 48 & 114.300 & 33 \\
\hline \multirow[t]{2}{*}{9} & -168.380 & 27 & -10.667 & 18 & -265.830 & 31 & 9.0853 & 44 \\
\hline & -18.385 & 23 & -54.920 & 19 & -35.599 & 34 & -45.092 & 35 \\
\hline \multirow[t]{2}{*}{10} & -171.830 & 14 & 24.229 & 21 & -498.710 & 48 & 33.976 & 33 \\
\hline & 6.8877 & 21 & -26.809 & 26 & 13.859 & 40 & -60.036 & 31 \\
\hline
\end{tabular}

in red. Mean $R^{2}$ values for the goodness of fit of estimated stiffness matrices to measured force data were 0.90 at baseline and 0.92 post-VGF training. In contrast to subjects receiving $\mathrm{EF}_{\text {strong }}$ training, no consistent changes in stiffness are seen. Figure $5 B$ shows mean stiffness matrix elements collapsed

A
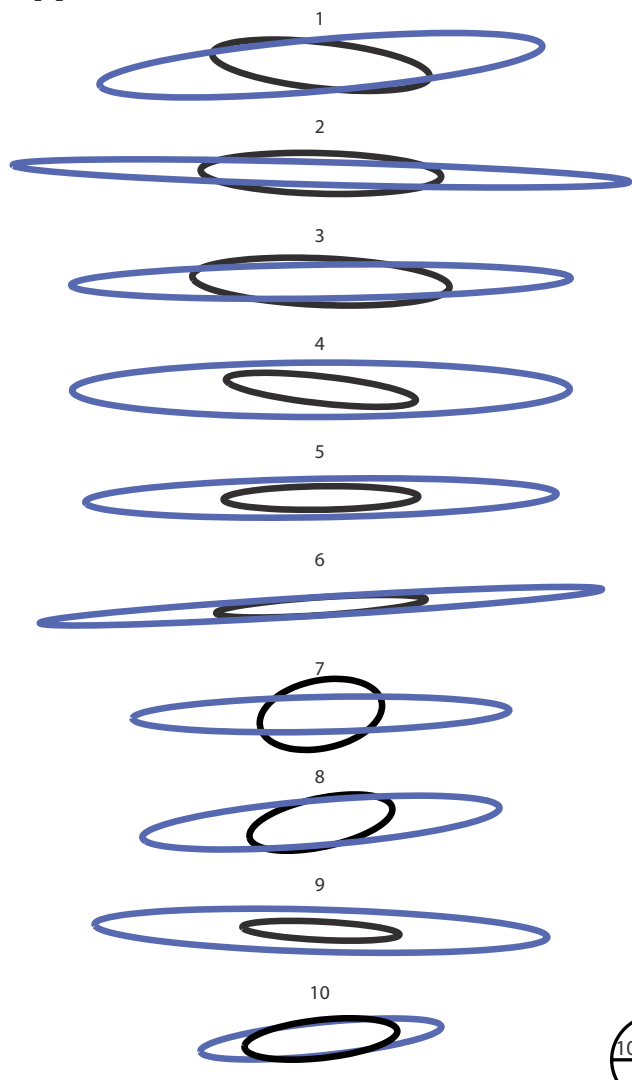

B

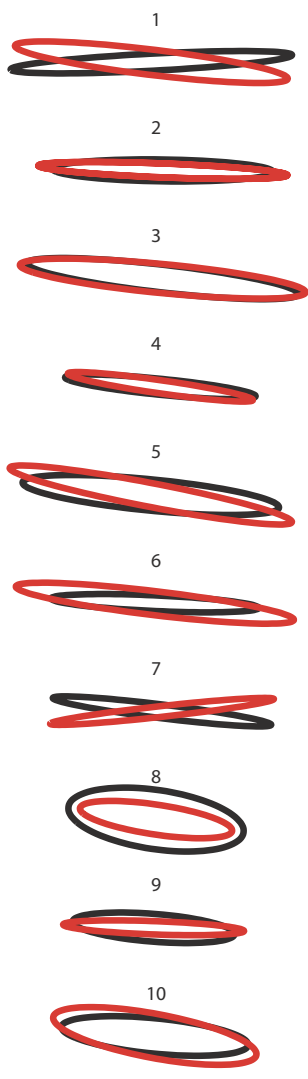

FIG. 4. Stiffness ellipses. Stiffness ellipses for subjects in all groups: $\mathrm{EF}_{\text {strong }}(n=10$, blue) and $\operatorname{VGF}(n=10$, red). Ellipses in black represent baseline stiffness for each subject; colored ellipses represent stiffness at the end of the respective training condition. 




FIG. 5. Stiffness matrices. A: stiffness components are shown averaged across $\mathrm{EF}_{\text {strong }}$ subjects at baseline (shown in black) and after adaptation (in blue). $B$ : VGF subjects at baseline (black) and after adaptation (red). $C: \mathrm{EF}_{\text {weak }}$ subjects at baseline (black) and after adaptation (green). Vertical bars represent $1 \mathrm{SE}$. although VGF subjects successfully reduced lateral movement variability, stiffness did not change.

To further investigate the nature of the observed increased movement precision in the visual gain condition we tested whether movements were adapted using on-line feedback control and/or changes to feedforward motor planning. To investigate changes in on-line feedback control, we examined movement precision in visual-gain catch trials, in which the cursor indicating limb position was extinguished throughout the movement. If subjects were using on-line visual feedback control to improve precision in the visual gain condition, then in catch trials in which vision was removed, movement precision ought to decrease. No statistically reliable differences in $\mathrm{mPD}$ were observed between catch trial without vision $($ mean $=5.7, \mathrm{SD}=1.2)$ and trials with vision $[$ mean $=5.9$, $\mathrm{SD}=1.1 ; F(1,18)=0.13 ; P=0.7]$. This result suggests that on-line feedback control was not a significant contributing factor to changes in movement precision.

To test for potential changes in feedforward motor planning as a result of training in the visual gain field, we examined the variability of the initial portion of movement. For each movement we measured the direction of a vector between hand position at movement start and hand position at peak tangential acceleration. This initial portion of movement is presumably determined by the initial movement plan and is not affected by on-line feedback control. The SD of the initial movement vector was significantly reduced after training in the visual gain field (mean $=1.67, \mathrm{SD}=0.53$ ) compared with the baseline condition $[$ mean $=2.36, \mathrm{SD}=0.80 ; F(1,18)=5.14 ; P=$ $0.036]$. This supports the idea that improvements in precision in the visual gain field were mediated by movement planning mechanisms, not by on-line feedback control. We also compared movement precision in baseline catch trials to catch trials in the VGF. Since visual feedback was eliminated during catch trials, any changes in movement precision cannot be attributed to on-line visual feedback control, but ought rather to depend on movement planning. Catch-trial $\mathrm{mPD}$ was significantly reduced in the VGF (mean $=2.74, \mathrm{SD}=0.74)$ compared with the baseline condition [mean $=3.40, \mathrm{SD}=1.3 ; F(1,18)=$ 5.18; $P=0.046]$. Finally, we also examined the number of lateral direction changes in baseline catch trials compared with VGF catch trials and found no significant difference $[F(1,18)=0.352 ; P=0.57]$. Taken together these analyses suggest that improvements to movement accuracy in the visual gain field were mediated not by on-line feedback control, but by feedforward movement planning mechanisms.

\section{Experiment 3: elastic field condition matched for kinematic error to the visual gain condition}

It can be seen in Fig. 3 that the magnitude of the initial perturbation in the $\mathrm{EF}_{\text {strong }}$ field was larger than that in the VGF field. Thus it is possible that no changes in stiffness were observed in the VGF group because the visual perturbation was not large enough. To rule this out, we tested a third group of subjects $(n=5)$ who were trained in a weaker divergent field $\left(\mathrm{EF}_{\text {weak }}\right)$. The magnitude of the elastic coefficient $(125 \mathrm{~N} / \mathrm{m})$ was chosen to match the magnitude of the initial perturbation in movement curvature to that observed in the VGF field. No significant difference was observed between $\mathrm{MPD}$ in the first 
TABLE 2. Stiffness matrices before and after training in a visual gain field (VGF)

\begin{tabular}{|c|c|c|c|c|c|c|c|c|}
\hline \multirow{3}{*}{ Subject } & & & \multicolumn{2}{|c|}{ Format for Entries } & \multirow{3}{*}{\multicolumn{3}{|c|}{ VGF }} & \\
\hline & & & $K_{x, x} \pm \mathrm{SE}$ & $K_{x, y} \pm \mathrm{SE}$ & & & & \\
\hline & & & $K_{y, x} \pm \mathrm{SE}$ & $K_{y, y} \pm \mathrm{SE}$ & & & & \\
\hline \multirow[t]{2}{*}{1} & -306.610 & 37 & 23.782 & 39 & -286.400 & 23 & 83.420 & 17 \\
\hline & -21.646 & 23 & -22.041 & 28 & 33.652 & 11 & -46.619 & 15 \\
\hline 2 & -232.490 & 13 & 62.716 & 17 & -255.640 & 10 & 93.436 & 22 \\
\hline 3 & 26.397 & 29 & -50.604 & 36 & 16.730 & 15 & -74.459 & 41 \\
\hline \multirow[t]{2}{*}{4} & -199.610 & 22 & 59.338 & 25 & -197.660 & 20 & 43.623 & 37 \\
\hline & 18.541 & 26 & -28.709 & 26 & 31.692 & 14 & -25.865 & 41 \\
\hline \multirow[t]{2}{*}{5} & -237.390 & 20 & 61.819 & 44 & -285.840 & 19 & 115.930 & 28 \\
\hline & 23.802 & 20 & -49.996 & 20 & 56.402 & 18 & -59.138 & 28 \\
\hline 6 & -170.440 & 14 & 24.160 & 29 & -214.480 & 20 & 85.030 & 25 \\
\hline \multirow[t]{2}{*}{9} & -173.730 & 14 & 34.029 & 18 & -186.350 & 17 & 66.282 & 23 \\
\hline & 9.3762 & 16 & -41.246 & 20 & 2.2754 & 19 & -20.348 & 20 \\
\hline \multirow[t]{2}{*}{10} & -173.040 & 15 & 108.030 & 30 & -192.720 & 24 & 110.620 & 27 \\
\hline & -5.3094 & 16 & -54.597 & 22 & 12.642 & 16 & -78.945 & 25 \\
\hline
\end{tabular}

movements in the VGF and $\mathrm{EF}_{\text {weak }}$ conditions $[F(1,13)=0.95$; $P=0.35]$. It should be noted that we also experimented with larger visual gains (e.g., 4.0) but we found that subjects tended to ignore the visual feedback when it was perturbed to such a large extent.

Subjects were initially perturbed by the weaker force field relative to baseline and were able to adapt and reduce the magnitude of positional deviations following training. Figure $3 B$ shows $\mathrm{mPD}$ as a function of training in the $\mathrm{EF}_{\text {weak }}$ field. For the first trial of exposure in the $\mathrm{EF}_{\text {weak }}$ field, $\mathrm{mPD}$ was increased to $11.6 \pm 7.2 \mathrm{~mm}$ compared with baseline of $4.1 \pm$ $1.4 \mathrm{~mm}$ and, by the end of training, mPD was reduced to $5.6 \pm$ $2.4 \mathrm{~mm}$.

Table 3 shows stiffness matrices calculated for $\mathrm{EF}_{\text {weak }}$ subjects. Mean $R^{2}$ values for goodness of fit of stiffness matrices to measured force data were 0.87 at baseline and 0.92 after training in $\mathrm{EF}_{\text {weak }}$. Consistent increases in the lateral stiffness element $K_{x, x}$ can be seen for all subjects. Figure $5 C$ shows mean values of the stiffness matrix across subjects. Baseline stiffness is shown in black. A MANOVA performed on all four stiffness matrix elements reached significance $[F(1,8)=5.53$; $P=0.044]$ and the sole statistical difference was found in $K_{x, x}$ $[F(1,8)=7.48 ; P=0.026]$; thus only stiffness in the lateral direction was increased during training in the divergent field $\mathrm{EF}_{\text {weak }}$. A $t$-test performed on the $K_{x, x}$ matrix elements showed a statistically significant difference between $\mathrm{EF}_{\text {strong }}$ and $\mathrm{EF}_{\text {weak }}[F(1,13)=15.6 ; P=0.002]$. Since $\mathrm{EF}_{\text {strong }}$ subjects increased their stiffness more than $\mathrm{EF}_{\text {weak }}$ subjects, adaptation was proportional to the environmental stability.

We performed the same analyses of catch-trial $\mathrm{mPD}$ and initial movement vector variability for $\mathrm{EF}_{\text {strong }}$ and $\mathrm{EF}_{\text {weak }}$ subjects, to determine whether EF training resulted in changes to feedforward commands comparable to those observed following VGF training. Unlike the VGF, we did not find statistically reliable changes in catch-trial $\mathrm{mPD}$ following $\mathrm{EF}$ training, for either group $\left[\mathrm{EF}_{\text {strong }}\right.$ baseline $=4.1 \pm 1.6 \mathrm{~mm}$; posttraining $=2.81 \pm 1.2 ; F(1,18)=1.98, P=0.21$;

TABLE 3. Stiffness matrices before and after training in a weaker elastic force field $\left(E F_{\text {weak }}\right)$

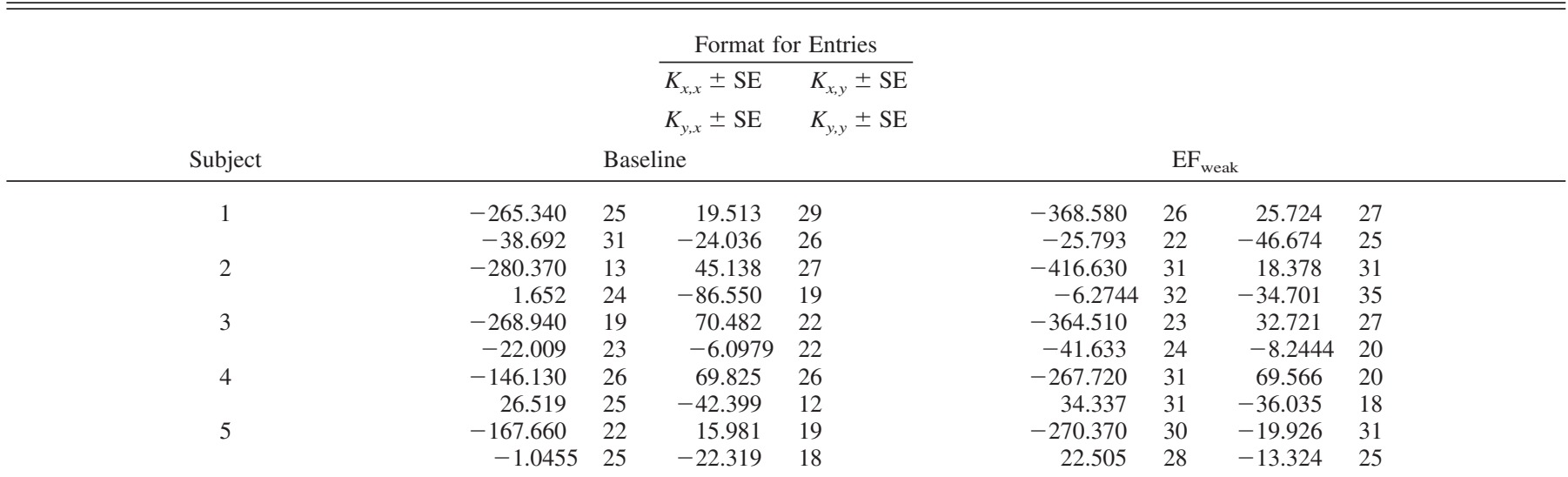


$\mathrm{EF}_{\text {weak }}=3.4 \pm 1.6 \mathrm{~mm}$; posttraining $=2.80, \mathrm{SD}=0.8 \mathrm{~mm}$; $F(1,8)=1.90, P=0.28]$.

To measure changes in the initial movement vector resulting from EF training, a set of zero force, no-vision catch trials were analyzed. We found no statistically reliable changes in the SD of these movement vectors for either EF group $\left[\mathrm{EF}_{\text {strong }}\right.$ baseline $=$ $1.60 \pm 1.3$; training $=2.1 \pm 1.3 ; F(1,18)=1.27 ; P=0.3 . \mathrm{EF}_{\text {weak }}$ baseline $=1.8 \pm 1.1 ;$ training $=1.9 \pm 1 ; F(1,8)=0.016 ; P=$ $0.9]$. Taken together, these analyses suggest that feedforward changes to movement planning did not occur for EF subjects.

We also compared force signal variability during stiffness perturbation trials (250-300 ms after perturbation onset) between VGF and EF subjects. Modulation of voluntary or involuntary responses could result in an increased SD of the force signal. No statistically reliable differences were found between $\mathrm{EF}_{\text {strong }}, \mathrm{VGF}$, and $\mathrm{EF}_{\text {weak }}$ groups in the $\mathrm{Y}$ force signal $\left[\mathrm{EF}_{\text {strong }} \mathrm{SD}=0.20 \pm 0.06 \mathrm{~N} ; \mathrm{VGF} \mathrm{SD}=0.23 \pm 0.04 \mathrm{~N}\right.$; $\left.\mathrm{EF}_{\text {weak }} \mathrm{SD}=0.25 \pm 0.07 \mathrm{~N} ; F(2,22)=1.60 ; P=0.225\right]$. We did find a small but statistically reliable difference in the SD of the force signal in the $\mathrm{X}$ direction for $\mathrm{EF}_{\text {strong }}$ subjects [SD = $0.48 \pm 0.13 \mathrm{~N} ; F(2,22)=8.49 ; P=0.002]$ compared with both $\mathrm{EF}_{\text {weak }}(\mathrm{SD}=0.32 \pm 0.05 \mathrm{~N})$ and $\mathrm{VGF}(\mathrm{SD}=0.33 \pm$ $0.04 \mathrm{~N}$ ); no difference was found in $\mathrm{X}$ force variability between VGF and $\mathrm{EF}_{\text {weak }}[F(2,13)=0.025, P=0.87]$. This slight increase in $\mathrm{X}$ force signal variability for $\mathrm{EF}_{\text {strong }}$ subjects may indicate some role for voluntary or involuntary feedback mechanisms as a result of $\mathrm{EF}_{\text {strong }}$ training; alternatively, this increase in variability may simply result from the greater magnitude of force (increase in mean) measured in the $\mathrm{X}$ direction for these subjects.

\section{I S C U S S I O N}

We investigated the effects of a purely visual perturbation to assess the role of vision in stiffness control. Our results show that a novel visual task resulting in increased movement precision is not sufficient for the motor system's use of stiffness control. In this perturbing environment the nervous system uses other methods of motor adaptation to effect the observed increase in movement accuracy. Previous research has shown that the motor system can adapt limb stiffness for movements made in an unstable force environment (Burdet et al. 2001; Franklin et al. 2003, 2004, 2007a). In these studies, information about the environmental instability was available from both visual and proprioceptive signals. Our results imply that the increase in stiffness measured in the preceding studies may not be a response to the visually perceived error, but rather may depend on proprioceptive and somatosensory signals related to the forces perturbing the limb.

There are a number of implications of these results. First, our results show that changes to movement accuracy may be observed in the absence of changes in limb stiffness. Although limb stiffness and cocontraction have been linked to movement variability in other studies (e.g., Gribble et al. 2003; Lametti et al. 2007; Osu et al. 2004; Selen et al. 2006; Shiller et al. 2002) our results imply that movement accuracy and stiffness may be independently controlled in some tasks but not others. This should have important implications for models of motor control that include central control of both movement and cocontraction (Feldman 1986; Flash and Gurevich 1997; Gribble and Ostry 2000; Gribble et al. 1998; Kistemaker et al. 2007a,b).
Many previous studies of stiffness modulation have been conducted using unstable, divergent force fields (e.g., Franklin et al. 2007a). Although it may not be immediately clear why subjects would increase stiffness in response to a purely visual manipulation, it should be noted that several studies have been reported in which subjects modulate limb stiffness in response to changes in visual task constraints such as target size (Gribble et al. 2003; Osu et al. 2004; Selen et al. 2006). The lack of such changes in stiffness in the present study implies that for the task studied here, in which visual feedback was similar to that present in divergent force field tasks, the visual information was not sufficient to elicit changes in limb stiffness. The role of visual information in the neural control of limb stiffness seems to be rather task specific. The benefits of increasing stiffness in the context of divergent force field tasks may be more clearly defined. Indeed, stiffness increases may be the only practical option available to the nervous system for increasing performance in an unstable force environment.

In a recent article, Franklin et al. (2007b) reported that subjects are capable of learning to reach in novel force fields and, in addition, are capable of modulating limb stiffness, in the absence of visual feedback. This is an important demonstration of the influential role of proprioceptive and somatosensory signals for limb stiffness modulation, but does not address the potential role of visual signals, when present. Although stiffness modulation can occur in the absence of vision, this does not preclude the possibility that visual signals (especially signals related to a perturbation) may be utilized in determining neural control signals for limb stiffness. The present study thus addresses the complementary question of whether perturbation-related visual signals, when present, play a role in the neural control of limb stiffness. In contrast to other tasks involving visual manipulations of various types (Gribble et al. 2003; Osu et al. 2004; Selen et al. 2006), in the task studied here, visual signals alone are not sufficient to elicit changes in limb stiffness.

It has been recently shown that stiffness control is used in parallel with adaptation to novel dynamics (Franklin et al. 2003; Thoroughman and Shadmehr 1999). Our results show that increases in movement accuracy can occur in the absence of stiffness change, which in turn suggests that other motor strategies, such as the refinement of movement control signals, can be used independent of stiffness control. This is consistent with the idea that the motor system generates independent control signals for stiffness and for movement (Feldman 1986; Flanagan et al. 1993; Gribble and Ostry 1998; Gribble et al. 1998).

The specific mechanism by which the motor system augments movement accuracy, in the absence of stiffness control, is unclear, although the data from the present studies allow us to discuss a number of possibilities. It may be that the increased visual information about movement variability, available in the visual gain condition, allowed for the refinement of control signals for movement. Indeed, follow-up analyses of the data from experiment 2 (see RESULTS) support the idea that improvements in movement precision in the visual gain field were based on changes to the precision of feedforward control signals, rather than changes in on-line feedback control.

The idea that movement control signals are modified in response to visual perturbations of movement trajectories is supported by previous work by Flanagan and Rao (1995). In their experiment, arm movement trajectories were displayed 
visually to subjects in a shoulder-elbow joint-space coordinate system rather than in a Cartesian hand-space coordinate system. With practice subjects learned to produce movements that were straight in the visually presented joint space, despite the fact that the corresponding arm movements were in fact highly curvilinear. In our study, the increased perception of movement curvature may have resulted in a similar process of adaptation in which subjects were able to utilize the visually amplified information about lateral hand position to further refine feedforward control signals for movement.

Our findings are important in relation to other investigations of stiffness control in the absence of external forces. Recent work has demonstrated increases in stiffness at the end of movement to match accuracy constraints such as target size or shape (Gribble et al. 2003; Lametti et al. 2007; Osu et al. 2004; Selen et al. 2006b); however, these investigations have focused on changes at movement end. Lametti et al. (2007) showed that higher stiffness levels at the end of movement were associated with greater endpoint accuracy and this relationship was independent of movement direction. Gribble et al. (2003) showed that when reaching to visual targets, subjects increased tonic electromyographic (EMG) levels at shoulder and elbow muscles at the end of movements for small targets compared with larger targets. Osu et al. (2004) observed greater levels of EMG activity in elbow muscles that corresponded with lower movement variability, but notably, differences in variability were found only at movement end. Selen et al. (2006a) reported that elbow-joint stiffness during the final portion of single-joint elbow movements was correlated with endpoint accuracy. In contrast, the current study focused on assessing stiffness during movement. We observed no changes in stiffness during the middle portion of movement in response to adaptation to a visual perturbation. It may be that in the absence of external perturbing forces, stiffness control is used conservatively in select circumstances, such as in endpoint control (Selen et al. 2006b).

Stiffness values estimated in the current study are comparable to those reported in the literature for human planar arm movements. A broad range of stiffness estimates have been reported for longitudinal (outward) multijoint reaching movements. Frolov et al. (2006) reported anisotropic stiffness ellipses with maximal stiffness in the transverse direction. Mah (2001) reported stiffness values that varied greatly depending on the point at which stiffness measurements were performed. Stiffness values reported in the current study are similar to those reported by Frolov et al. (2006) and Mah (2001), although stiffness magnitudes here are generally higher. This may reflect the higher movement speeds in the current study (Gerritsen et al. 1998; Suzuki et al. 2001). Stiffness estimates reported by Burdet et al. (2001) and Franklin et al. (2003) are comparable to those reported in the current study. Stiffness in the lateral direction $\left(K_{x, x}\right)$ ranged from about 150 to $400 \mathrm{~N} / \mathrm{m}$ in both Burdet et al. (2001) and Franklin et al. (2003), whereas in this study $K_{x, x}$ ranged from 146 to $307 \mathrm{~N} / \mathrm{m}$. Estimated stiffness along the axis of movement $\left(K_{y, y}\right)$ was higher in these studies than in the current study, with values ranging from about 180 to $300 \mathrm{~N} / \mathrm{m}$ in comparison to $16-93 \mathrm{~N} / \mathrm{m}$ in this study. Again, this may reflect differences in movement speed between the current study and previous reports. Darainy et al. (2007) reported anisotropic stiffness estimates during outward reaches, similar to Frolov et al. (2006). We used the same movement distances and speeds of Darainy et al. (2007) and we found very similar magnitudes of limb stiffness. The average values of the four elements of the hand stiffness matrix were $[-208.2,60.0 ; 39.2,-47.2] \mathrm{N} / \mathrm{m}$ in Darainy et al. (2007), compared with $[-206.2,69.2 ; 21.7,-50.9] \mathrm{N} / \mathrm{m}$ in this study. It should be noted that although in the present study we used only three perturbations per direction, our estimates of stiffness were similar to those reported by Darainy et al. (2007), who used six perturbations per direction.

The various methodological differences in measuring multijoint stiffness likely contribute to the different stiffness estimates reported in the literature. Several previous studies used force perturbations (Frolov et al. 2006; Gomi and Kawato 1997; Mah 2001), which require estimation of inertia and viscosity parameters in addition to stiffness. In contrast, other studies (including the current study) have used servo-controlled position perturbations, which require only estimation of stiffness (Burdet et al. 2001; Darainy et al. 2007; Franklin et al. 2003). Some studies used a fiberglass cuff to attach the arm to the manipulandum (Burdet et al. 2001; Franklin et al. 2003; Gomi and Kawato 1997), whereas others required subjects to grasp a handle (Darainy et al. 2007; Mah 2001). Another methodological variable that could affect stiffness measurements is the frequency at which stiffness measurement perturbations were applied within the set of movements. The rate at which perturbation trials are performed balances the length of time required to estimate stiffness with the potential for motor adaptation, since the perturbations themselves could result in behavioral changes. In this study, test perturbations were applied at a frequency of $20 \%$ (the same as in Darainy et al. 2007). It should be noted that others have applied perturbations much more frequently: at 40\% (Mah 2001) and also 50\% (Burdet et al. 2001; Franklin et al. 2003).

It should be noted that one additional difference between the visual gain field and unstable elastic field-tested here and by Burdet et al. (2001) — is that the forces in the elastic field are unstable. That is to say, small errors lead to increased forces on the hand, which in turn lead to larger errors, and so on. No such instability was present in the VGF experiments. Despite this, the learning rate in the VGF was comparable to that in the elastic field. The specific role of stability and the corresponding need for appropriate compensation may be assessed in future experiments. It should also be noted that the lack of stiffness modulation in the VGF task studied here does not rule out the possibility that, in other tasks, visual signals may play a more significant role in driving stiffness adaptation.

In summary, we have shown that although subjects selectively increase limb stiffness in response to an unstable elastic force field applied to the limb, they do not modulate stiffness in response to a similar, purely visual perturbation, despite successfully increasing movement accuracy. Although stiffness control has been linked to movement accuracy in previous studies, our results imply that stiffness and movement variability may be independently controlled in a task-specific manner.

\section{A P PENDIX A}

The equation of motion of the two-joint planar arm can be expressed as

$$
I(q) \ddot{q}+C(\dot{q}, q)=\tau(\dot{q}, q, t)+\tau_{e x t}
$$

where $q$ is the angular position of the shoulder and the elbow. The arm components (inertial $I$ and Coriolis-centrifugal $C$ ) are equal to the 
muscle torque $t$, and any external torque $t_{\text {ext }}$ (in this case, the external torque is applied by the torque motors in the robotic linkage). For small deviations, this equation can be linearized

$$
I \partial \ddot{q}+\frac{\partial I \ddot{q}}{\partial q} \partial q+\frac{\partial C}{\partial \dot{q}} \partial \dot{q}+\frac{\partial C}{\partial q} \partial q=\frac{\partial \tau}{\partial \dot{q}} \partial \dot{q}+\frac{\partial \tau}{\partial q} \partial q+\delta \tau_{\text {ext }}
$$

Consider a small, pure positional deviation, where

$$
\partial \ddot{q}=\partial \dot{q}=0
$$

This linearized equation reduces to

$$
\left(\frac{\partial I \ddot{q}}{\partial q}+\frac{\partial C}{\partial q}\right) \partial q-\frac{\partial \tau}{\partial q} \partial q=\tau_{e x t}
$$

Thus torque at the joints from a small perturbation is equal to the sum of a dynamics component (inertial and Coriolis-centrifugal) and the joint stiffness, $-(\partial \tau / \partial q) \partial q$. This relationship can be translated to hand (i.e., Cartesian) coordinates and forces using the Jacobian matrix, leaving

$$
\Delta F=-\frac{\mathrm{d} F_{a}}{\mathrm{~d} x} \Delta x+K_{x} \Delta x
$$

Thus the stiffness matrix $K_{x}$ is a function of three terms: the change in force $\Delta F$, change in position $\Delta x$, and $\mathrm{d} F_{a} / \mathrm{d} x$, the contribution of dynamics to restoring forces. This dynamics component $\left(\mathrm{d} F_{a} / \mathrm{d} x\right) \Delta x$ may be computed numerically (Burdet and Osu 1999) on a per subject basis, using measurements of limb-segment lengths and estimates of limb-segment masses and moments of inertia from anthropometric data (Winter 2005). Note that change in force $\Delta F$ is equal to the recorded force minus an estimate of force at the handle that would be exerted if there was no perturbation

$$
\Delta F=F_{a c t}-F_{e s t}
$$

where $F_{\text {act }}$ can be read from the force transducer and $F_{\text {est }}$ must be estimated. Likewise, the perturbation distance $\Delta x$ is

$$
\Delta x=x_{a c t}-x_{e s t}
$$

where $x_{a c t}$ is the instanteneous position of the hand and $x_{\text {est }}$ is an estimate of the position of the hand that would be expected if there had been no perturbation.

After accounting for $\left(\mathrm{d} F_{a} / \mathrm{d} x\right) \Delta x$

$$
\Delta F=K_{x} \Delta x
$$

where the stiffness matrix $K_{x}$ is calculated by linear regression. In this study, perturbation of the hand to the desired location was realized in $100 \mathrm{~ms}$ using a minimum-jerk trajectory to maximize smoothness (Burdet et al. 2000), and estimates of restoring force were made following another $150 \mathrm{~ms}$ to allow any position oscillations to cease, and force signals to stabilize.

Estimates of the $\mathrm{X}$ and $\mathrm{Y}$ components of hand position were made using autoregression (AR). AR models compute the next output of a time series from a linear weighting of the past $n$ time samples. For an estimate $\hat{x}_{t}$ of the position signal $x$ at time $t$

$$
\hat{x}_{t}=\sum_{i=1}^{n} r_{i} x_{t-i}+\varepsilon
$$

where $n$ is the order of the model, $r_{i}$ for $i=1: n$ are the AR coefficients, and $\varepsilon$ is the residual. To calculate all $r$, the means of both signals were subtracted and a standard least-squares method was used in MATLAB (The MathWorks). Thus the on-line estimation for $x$ was

$$
\hat{x}_{t}=\bar{x}+\sum_{i=1}^{n} r_{i} x_{t-i}
$$

where $\bar{x}$ is the mean of $x$ over the prediction window and $x_{t-i}$ is the zero-mean adjusted signal. Selection of both the order and sampling rate of the AR model was done to maximize model fit. Previously an eight-order model was used at $75 \mathrm{~Hz}$ (Darainy et al. 2007); here we used the same parameters. Thus predictions of position were based on the preceding $100 \mathrm{~ms}$ of movement. The middle $500 \mathrm{~ms}$ of the movement was predicted using the AR model, centered midway between the start and end targets.

Forty baseline movements were recorded for each subject and used to calculate the AR coefficients in advance of stiffness measurement. These movements were performed following a familiarization and training period not shorter than 100 movements, after which time consistently accurate and well-timed movements were observed.

\section{Cross-validation tests of the AR model}

Prediction error of the AR model was determined by cross-validation using unperturbed movements. The AR model was used to predict a 50-ms segment of hand position signals (located at the point in the trajectory at which stiffness would have been estimated had these been perturbed movements), for a set of 40 unperturbed movements (for which the entire trajectory was known). The mean prediction error of the AR model for both $x$ and $y$ position signals was small; mean $\pm \mathrm{SD}$ of the prediction errors in $[\mathrm{X}, \mathrm{Y}]$ were $[-0.503 \pm 2.4$, $0.33 \pm 4.1] \mathrm{mm}$. Mean absolute prediction error at the end of the stiffness estimation window (after $300 \mathrm{~ms}$ ) was $[1.8 \pm 1.5,4.5 \pm$ $2.18 \mathrm{~mm}$, resulting in mean absolute tangential error of $4.9 \mathrm{~mm}$. Positional prediction errors here compare favorably with other reported prediction errors of about $1 \mathrm{~cm}$ (Burdet et al. 2000). Moreover, we observed no particular bias in the estimates of hand position that may result in biased estimates of stiffness matrix elements.

An estimation of the unperturbed force signal $F_{\text {est }}$ was made using simple correlation. The estimated force signal over the perturbation window was chosen from the set of 40 baseline unperturbed movements as the force signal most correlated with the force signal for perturbed movements during the time window $50 \mathrm{~ms}$ before and 150 ms after perturbation end. Cross-validation using this method resulted in very accurate estimates: mean $\pm \mathrm{SD}$ force prediction errors in $\left[F_{x}\right.$, $\left.F_{y}\right]$ were $[0.13 \pm 0.21,-0.21 \pm 0.25] \mathrm{N}$; mean absolute force errors at the end of the $300-\mathrm{ms}$ prediction window were $[0.25 \pm 0.16$, $0.37 \pm 0.21] \mathrm{N}$. An AR model was also tested for force estimation, but it did not perform as well as the correlation method. The AR force model was sensitive to biases in the force transducer that changed between experimental blocks (i.e., each 40 movement set).

\section{A PPENDIX B}

\section{Force signal measurement controls}

To control for the possibility that voluntary feedback affected estimations of restoring force, precautionary measures were taken to determine that force signals were stable, not highly sensitive to the selected time window, and not contaminated by voluntary reactions. First, control experiments were performed in which subjects were explicitly instructed to attempt to intervene after a perturbation, contrary to directions provided to experimental subjects. Voluntary reaction responses were clearly visible in perturbed trials, characterized by a continuous increase in restoring force. This allowed the identification of any trials suspected of intervention. SDs of the force signal $>1 \mathrm{~N}$ during a window 250-300 ms after perturbation onset were identified as intervened trials and removed, which applied to $<2 \%$ of trials. 
Second, the stability of force signals was measured over the estimation time window. Stable force signals (i.e., no sudden or continuous changes in force) would also argue against voluntary intervention. During the force estimation window on perturbed trials, force signal SD was measured to be $[0.28,0.21] \mathrm{N}$. In comparison, unperturbed movements show variability during this time window of $[0.16,0.17] \mathrm{N}$, which shows that the variability during stiffness estimates is somewhat larger, but close to the unperturbed condition, and still quite small. Force signal SD was also measured in an adjacent time window (300-350 ms) and was found to be $[0.35,0.25] \mathrm{N}$; thus signal variability is relatively small.

Another way to increase our confidence in stiffness estimates was to perform stiffness measurements in both time windows (250-300 and $300-350 \mathrm{~ms}$ ) and compare the results. Stiffness measurements thus were performed at an adjacent time window (300-350 ms) and close agreement between the two stiffness calculations would suggest that stiffness matrix measurements are stable over a period of $100 \mathrm{~ms}$. Stiffness matrices for the two adjacent windows were [-206.2, 69.2; $21.7,-50.9]$ and $[-210.2,45.1 ; 8.9,-54.3] \mathrm{N} / \mathrm{m}$. These analyses show that force signals during this time window are relatively stable, especially for matrix components $K_{x, x}$ and $K_{y, y}$, and stiffness matrix estimates are very similar over a $100-\mathrm{ms}$ time window. Combined, the observed small change in force signals during the estimation window and consistent stiffness estimations for two consecutive windows spanning $100 \mathrm{~ms}$ suggest that measurements of force are reliable and unlikely to reflect effects of voluntary correction.

\section{Stiffness matrix sensitivity analysis: sensitivity to estimation error for restoring forces}

To determine the sensitivity of matrix estimates to errors in force estimation, stiffness matrices were recalculated by adding Gaussian noise to all $24 \mathrm{~d} F$ measurements, and recomputing the stiffness matrix. The $\mathrm{d} F$ values were calculated for two adjacent time windows (250-300 and 300-350 ms) and these data were used to calculate mean and SD of signal variance. This difference between means of two adjacent time windows is some representative measure of the signal variability during the estimation period. Means and SDs were calculated separately for each of the eight pulse directions, to capture direction-dependent variability in force measurements. Stiffness matrices were recomputed 1,000 times with random noise added to all 24 $\mathrm{d} F$ values recorded for one stiffness estimate, randomly chosen. The recomputed matrices resulted in SDs of $[17,14 ; 14,11] \mathrm{N} / \mathrm{m}$, showing that the effects of variations in recorded restoring forces to the overall stiffness matrix are relatively small.

\section{Stiffness matrix sensitivity analysis: sensitivity to estimation error of position servos}

To determine the sensitivity of matrix estimates to errors of the position estimation, stiffness matrices were recalculated by adding Gaussian noise to position estimates. The $\mathrm{d} P$ values were recalculated by adding the mean \pm 1 SD values obtained from cross-validation (see earlier text), where errors in the $\mathrm{X}$ and $\mathrm{Y}$ position prediction were found to be $-0.503 \pm 2.4$ and $0.33 \pm 4.1 \mathrm{~mm}$, respectively. Again, these recomputed matrices resulted in SDs of $[15,10 ; 15,13] \mathrm{N} / \mathrm{m}$, which shows that the variations in the positional prediction do not greatly affect the overall stiffness estimates.

\section{A C K N O W L E D G M EN T S}

We thank M. Darainy, D. Ostry, and D. Kistemaker for helpful comments.

\section{G R A N T S}

This work was supported by National Institutes of Health, Natural Sciences and Engineering Research Council of Canada, and Canadian Institutes of Health Research.

\section{REFERENCES}

Burdet E, Osu R. Development of a New Method for Identifying Muscle Stiffness During Human Arm Movements. Technical Report 1-21. Japan: Kawato Dynamic Brain Project, Exploratory Research for Advanced Technology, Japan Science and Technology Agency, 1999.

Burdet E, Osu R, Franklin DW, Milner TE, Kawato M. The central nervous system stabilizes unstable dynamics by learning optimal impedance. Nature 414: 446-449, 2001.

Burdet E, Osu R, Franklin DW, Yoshioka T, Milner TE, Kawato M. A method for measuring endpoint stiffness during multi-joint arm movements. J Biomech 33: 1705-1709, 2000.

Darainy M, Malfait N, Gribble PL, Towhidkhah F, Ostry DJ. Learning to control arm stiffness under static conditions. J Neurophysiol 92: 3344-3350, 2004.

Darainy M, Malfait N, Towhidkhah F, Ostry DJ. Transfer and durability of acquired patterns of human arm stiffness. Exp Brain Res 170: 227-237, 2006.

Darainy M, Towhidkhah F, Ostry DJ. Control of hand impedance under static conditions and during reaching movement. J Neurophysiol 97: 2676-2685, 2007.

Feldman AG. Once more on the equilibrium-point hypothesis (lambda model) for motor control. J Mot Behav 18: 17-54, 1986.

Flanagan JR, Ostry DJ, Feldman AG. Control of trajectory modifications in target-directed reaching. J Mot Behav 25: 140-152, 1993.

Flanagan JR, Rao AK. Trajectory adaptation to a nonlinear visuomotor transformation: evidence of motion planning in visually perceived space. J Neurophysiol 74: 2174-2178, 1995.

Flash T, Gurevich I. Arm trajectory generation and stiffness control during motor adaptation to external loads. In: Self-Organization, Computational Maps and Motor Control, edited by Morasso PG, Sanguinetti V. Amsterdam: Elsevier Science, 1997, p. 423-482.

Franklin DW, Burdet E, Osu R, Kawato M, Milner TE. Functional significance of stiffness in adaptation of multijoint arm movements to stable and unstable dynamics. Exp Brain Res 151: 145-157, 2003.

Franklin DW, Liaw G, Milner TE, Osu R, Burdet E, Kawato M. Endpoint stiffness of the arm is directionally tuned to instability in the environment. J Neurosci 27: 7705-7716, 2007a.

Franklin DW, Osu R, Burdet E, Kawato M. Visual feedback is not necessary for the learning of novel dynamics. PLOS ONE 2: e1336, $2007 \mathrm{~b}$.

Franklin DW, So U, Kawato M, Milner TE. Impedance control balances stability with metabolically costly muscle activation. J Neurophysiol 92 : 3097-3105, 2004.

Frolov AA, Prokopenko RA, Dufosse M, Ouezdou FB. Adjustment of the human arm viscoelastic properties to the direction of reaching. Biol Cybern 94: 97-109, 2006.

Gerritsen KGM, van den Bogert AJ, Hulliger M, Zernicke RF. Intrinsic muscle properties facilitate locomotor control: a computer simulation study. Motor Control 2: 206-220, 1998.

Gomi H, Kawato M. Equilibrium-point control hypothesis examined by measured arm stiffness during multijoint movement. Science 272: 117-120, 1996.

Gomi H, Kawato M. Human arm stiffness and equilibrium-point trajectory during multi-joint movement. Biol Cybern 76: 163-171, 1997.

Gribble PL, Mullin LI, Cothros N, Mattar A. Role of cocontraction in arm movement accuracy. J Neurophysiol 89: 2396-2405, 2003.

Gribble PL, Ostry DJ. Independent coactivation of shoulder and elbow muscles. Exp Brain Res 123: 355-360, 1998.

Gribble PL, Ostry DJ. Compensation for loads during arm movements using equilibrium-point control. Exp Brain Res 135: 474-482, 2000.

Gribble PL, Ostry DJ, Sanguineti V, Laboissiere R. Are complex control signals required for human arm movement? J Neurophysiol 79: 1409-1424, 1998.

Hogan N. The mechanics of multi-joint posture and movement control. Biol Cybern 52: 315-331, 1985.

Kistemaker DA, Van Soest AK, Bobbert MF. Equilibrium point control cannot be refuted by experimental reconstruction of equilibrium point trajectories. J Neurophysiol 98: 1075-1082, 2007a.

Kistemaker DA, Van Soest AJ, Bobbert MF. A model of open-loop control of equilibrium position and stiffness of the human elbow joint. Biol Cybern 96: 341-350, 2007b.

Lametti DR, Houle G, Ostry DJ. Control of movement variability and the regulation of limb impedance. J Neurophysiol 98: 3516-3524, 2007.

Mah CD. Spatial and temporal modulation of joint stiffness during multijoint movement. Exp Brain Res 136: 492-506, 2001. 
Osu R, Kamimura N, Iwasaki H, Nakano E, Harris CM, Wada Y, Kawato M. Optimal impedance control for task achievement in the presence of signal-dependent noise. J Neurophysiol 92: 1199-1215, 2004.

Seidler-Dobrin RD, He J, Stelmach GE. Coactivation to reduce variability in the elderly. Motor Control 4: 314-330, 1998.

Selen LP, Beek PJ, van Dieen JH. Impedance is modulated to meet accuracy demands during goal-directed arm movements. Exp Brain Res 172: 129-138, $2006 a$.

Selen LP, van Dieen JH, Beek PJ. Impedance modulation and feedback corrections in tracking targets of variable size and frequency. $J$ Neurophysiol 96: $2750-2759,2006 b$.
Shiller DM, Laboissiere R, Ostry DJ. Relationship between jaw stiffness and kinematic variability in speech. J Neurophysiol 88: 2329-2340, 2002 .

Suzuki M, Shiller DM, Gribble PL, Ostry DJ. Relationship between cocontraction, movement kinematics and phasic muscle activity in single-joint arm movement. Exp Brain Res 140: 171-181, 2001.

Thoroughman KA, Shadmehr R. Electromyographic correlates of learning an internal model of reaching movements. J Neurosci 19: 8573$8588,1999$.

Winter DA. Biomechanics and Motor Control of Human Movement. Hoboken, NJ: Wiley, 2005. 